\title{
High-throughput sequencing reveals miRNA effects on the primary and secondary production properties in long-term subcultured Taxus cells
}

\section{OPEN ACCESS}

Edited by:

Jun Yu,

Beijing Institute of Genomics, China

Reviewed by:

Hui Wang,

Natural Environmental Research

Council, UK

Shuhui Song,

Chinese Academic Science, China Ing-Feng Chang

National Taiwan University, Taiwan

${ }^{\star}$ Correspondence:

Chunhua Fu,

Department of Biotechnology, Institute

of Resource Biology and

Biotechnology, College of Life Science and Technology, Huazhong University of Science and Technology, No.1037 Luoyu Road, Wuhan, 430074, China fuch2003@126.com

${ }^{\dagger}$ These authors have contributed equally to this work.

Specialty section:

This article was submitted to Plant Genetics and Genomics,

a section of the journal

Frontiers in Plant Science

Received: 12 May 2015 Accepted: 22 July 2015

Published: 06 August 2015

Citation:

Zhang M, Dong Y, Nie L, Lu M, Fu C

Yu L (2015) High-throughput sequencing reveals miRNA effects on the primary and secondary production properties in long-term subcultured Taxus cells. Front. Plant Sci. 6:604. doi: 10.3389/fpls.2015.00604

\section{Meng Zhang ${ }^{1 \dagger}$, Yanshan Dong ${ }^{1 \dagger}$, Lin Nie ${ }^{1}$, Mingbo $\mathrm{Lu}^{1,2}$, Chunhua $\mathrm{Fu}^{1,2 *}$ and Longjiang $Y u^{1,2}$}

'Department of Biotechnology, Institute of Resource Biology and Biotechnology, College of Life Science and Technology, Huazhong University of Science and Technology, Wuhan, China, ${ }^{2}$ Key Laboratory of Molecular Biophysics Ministry of Education, College of Life Science and Technology, Huazhong University of Science and Technology, Wuhan, China

Plant-cell culture technology is a promising alternative for production of high-value secondary metabolites but is limited by the decreased metabolite production after long-term subculture. The goal of this study was to determine the effects of miRNAs on altered gene expression profiles during long-term subculture. Two Taxus cell lines, CA (subcultured for 10 years) and NA (subcultured for 6 months), were high-throughput sequenced at the mRNA and miRNA levels ${ }^{1}$. A total of 265 known (78.87\% of 336) and 221 novel $(79.78 \%$ of 277 ) miRNAs were differentially expressed. Furthermore, $67.17 \%$ of the known differentially expressed (DE) miRNAs (178) and $60.63 \%$ of the novel DE-miRNAs (134) were upregulated in NA. A total of 275 inverse-related miRNA/mRNA modules were identified by target prediction analysis. Functional annotation of the targets revealed that the high-ranking miRNA targets were those implicated in primary metabolism and abiotic or biotic signal transduction. For example, various genes for starch metabolism and oxidative phosphorylation were inversely related to the miRNA levels, thereby indicating that miRNAs have important roles in these pathways. Interestingly, only a few genes for secondary metabolism were inversely related to miRNA, thereby indicating that factors other than miRNA are present in the regulatory system. Moreover, miR8154 and miR5298b were upregulated miRNAs that targeted a mass of DE genes. The overexpression of these miRNAs in CA increased the genes of taxol, phenylpropanoid, and flavonoid biosynthesis, thereby suggesting their function as crucial factors that regulate the entire metabolic network during long-term subculture. Our current studies indicated that a positive conversion of production properties from secondary metabolism to primary metabolism occurred in long-term subcultured cells. miRNAs are important regulators in the upregulation of primary metabolism.

Keywords: plant-cell culture, long-term subculture, miRNA regulation, production properties conversion, Taxus chinensis

Abbreviations: DE, Differentially expressed; FW, fresh weight; DW, drought weight; DBIII, 10-Deacetylbaccatin III; DET, 10-deacetyl taxol; CEP, cephalomanine; ETOL, 7-epi taxol; BIII, baccatin III; qRT-PCR, quantitative Real Time-PCR; Tasy, taxadiene synthase; Dbat, 10-deacetylbaccatin III-10-O-acetyl transferaseferase; Pal, phenylalanine ammonia-lyase; Chs, chalcone synthase.

${ }^{1}$ The raw reads of mRNA- and miRNA-seq in this article were submitted to SRA with accession number SRR1339463, SRR1339474 and SRR1343578. 


\section{Introduction}

Most of the valuable natural products from plants are present at very low concentrations. For instance, only $1 \mathrm{~kg}$ of taxol is present in a thousand century-old Taxus trees. Direct isolation of these secondary metabolites from plant tissues was a rough way leading to an extinct exploration. Plant cell culture technology is a promising alternative for production of high-value secondary metabolites (Lila, 2005; Zhao and Verpoorte, 2007). However, several studies reported that a series of changes occurred in long-term subcultured cells, such that production became lower (Kolewe et al., 2008; Mustafa et al., 2011; Li et al., 2013). For instance, the long-term subcultured cells metabolically and morphologically differ; thus, the cell aggregates are heterogeneous subpopulations and individual cells within a culture accumulated in each product (Hall and Yeoman, 1987; Naill and Roberts, 2005; Kolewe et al., 2010). Patil et al. (2013) reported that the long-term subculture of Taxus cells formed numerous unevenly sized aggregates, thereby blocking taxol production. Moreover, the long-term subcultured cells frequently have epigenetic modifications (e.g., DNA methylation), and their cellular ploidy levels varied after long-term subculture (Baebler et al., 2005; Miguel and Marum, 2011). These observations implied that the conversion of longterm subcultures is related to a complicated and complex regulatory network. However, none of these reports showed the different gene expression profiles in long-term subcultured cells. The previous reports could not explain the regulatory mechanisms in detail.

In plants, miRNAs are important regulators of various activities, such as genome stability, development, and abiotic or biotic stress response (Jones-Rhoades and Bartel, 2004; Sunkar and Zhu, 2004; Lu et al., 2005; Mallory and Vaucheret, 2006; Vaucheret, 2006; Bartel, 2009; Xu et al., 2010). To date, several reports have combined mRNA-seq and miRNA-seq to elucidate the miRNA functions in complex problems in plants (Chen et al., 2012; Yang et al., 2012). He et al. (2013) determined the pathways involved in the rapid growth of developing culms in Moso bamboo. Pei et al. (2013) analyzed the miRNA and mRNA profiles in response to ethylene in rose petals during flower opening. Reynoso et al. (2013) revealed that polyribosomes selectively recruit mRNAs and miRNAs to respond to rhizobium infection in Medicago truncatula, thereby indicating the mechanism of nodule conformation. Therefore, the integrated analysis of miRNA and mRNA profiles could help us better understand the long-term subculture of cells.

Taxus sp. produces taxol, which is a widely used anticancer drug, but the taxol content is extremely low in plants (Howat et al., 2014). Given the problems of long-term subculture, current cell culture systems of paclitaxel production were not applicable for commercial use (Malik et al., 2011). Subcultured Taxus cells are a representative model for clarifying the conversion mechanism of long-term subculture. Therefore, two Taxus cell lines, namely, NA (newly separated and subcultured for 6 months with a high secondary metabolite biosynthesis ability) and CA (which is the control cell line being subcultured for 10 years with low secondary metabolite biosynthesis), were used to clarify the miRNA functions during the conversion of long-term subcultures.

\section{Results}

\section{NA had a Higher Production of Secondary Metabolites than CA}

The two cell lines, CA and NA, appeared to have different phenotypes. NA cells had slower growth and appeared bronze, whereas CA cells were beige. The secondary metabolite contents in CA and NA cell lines were significantly different. The flavonoid content in NA was $7.63 \mathrm{mg} / \mathrm{g}$ fresh weight (FW), which was 11.74 times higher than the $0.65 \mathrm{mg} / \mathrm{g} \mathrm{FW}$ in CA. The taxane production was also quantified. Results showed that the secondary metabolite levels were significantly higher in NA than in CA (Figure 1). According to our previous report (Song et al., 2014), the production of taxane can be represented by quantifying six compounds, including taxol. Each of the six compounds had significantly higher levels in NA (Figure 1), the amount of DBIII (10-deac), DET, CEP, and taxol was 3.12, 5.50, 5.18, and 1.88 times higher, respectively, than that in CA. BIII is an important precursor in taxane biosynthesis; notably, this compound could not be detected in CA, whereas the BIII content in NA was as high as $118 \mu \mathrm{g} / \mathrm{g}$ DW. All these results indicated that NA had stronger biosynthesis of secondary metabolites.

\section{Sequencing and Annotation of miRNA-seq}

A total of 19,998,242 and 19,686,076 raw reads were sequenced, with 19,843,361 and 19,558,835 high quality reads. Finally, $19,500,319$ and 19,212,618 clean reads were generated in CA and NA, respectively (Table S1). In the two samples, 21 nt-length and 24 nt-length small RNAs were the majority, although the 21 ntlength small RNA was the most dominant in NA and CA. In particular, the $21 \mathrm{nt}$-length small RNA was $48.83 \%$ of all the small RNAs in NA, with almost 1.8 times higher redundancy than CA (Figure 2).

Further annotation analysis divided these small RNA into miRNA, rRNA, snRNA, snoRNA, tRNA, and unannotated RNA by BLAST searches with public databases. In CA, 13,527 unique sequences $(0.65 \%)$ of the total 712,820 sequences $(3.66 \%)$ were annotated as miRNAs; 215 of which were identified as known miRNAs. In NA, 18,008 unique sequences $(0.61 \%)$ of the total

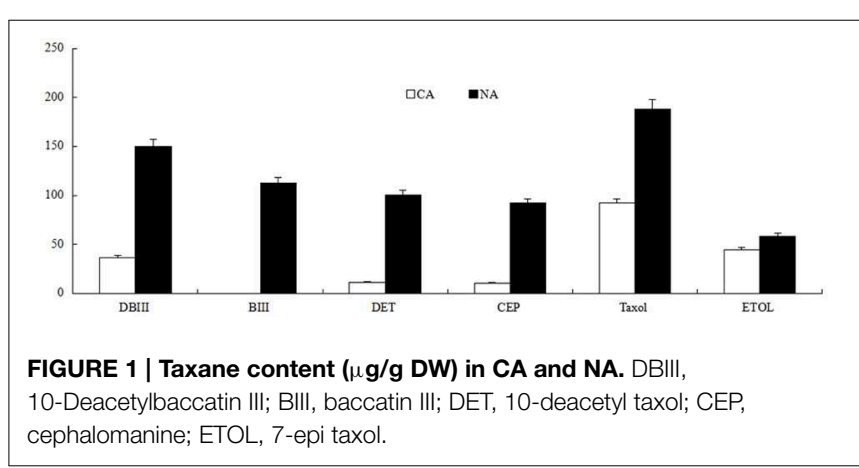


909,051 sequences (4.73\%) belonged to 286 miRNAs (Table 1). A total of 351 known miRNAs were identified, and 336 (95.73\%) were detected in both CA and NA.

The unannotated sRNAs were predicted with strict conditions to find novel miRNAs. After alignment with the simplified genome of Taxus baccata (Nystedt et al., 2013), only the miRNAs whose precursors could form a hairpin structure were considered to be miRNAs. Otherwise, these sRNAs were designated as pseudo-miRNAs. A total of 482 and 575 novel miRNAs were predicted in CA and NA, respectively. Only 277 novel miRNAs were detected in both CA and NA. Several variant structures of novel miRNAs are shown in Figure 3.

\section{Most miRNAs Changed their Expression Levels}

According to our data, most miRNAs changed their expression levels (Figure 4). Among the 336 known miRNAs in CA, miR2199, miR2916, miR397a, and miR164c were the most overrepresented, whereas novel_mir_281 and novel_mir_333 were the most overrepresented of the 277 novel miRNAs (Table S2). miR397 expression was significantly higher in NA, which was hardly detected with 0.01 TPM (transcript per million) in CA. Moreover, miR2199 and miR2916 were still highly abundant in NA. The novel miRNAs novel_mir_281 and novel_mir_333 were still the most overrepresented in NA. However, the abundance of novel_mir_933, novel_mir_266, and

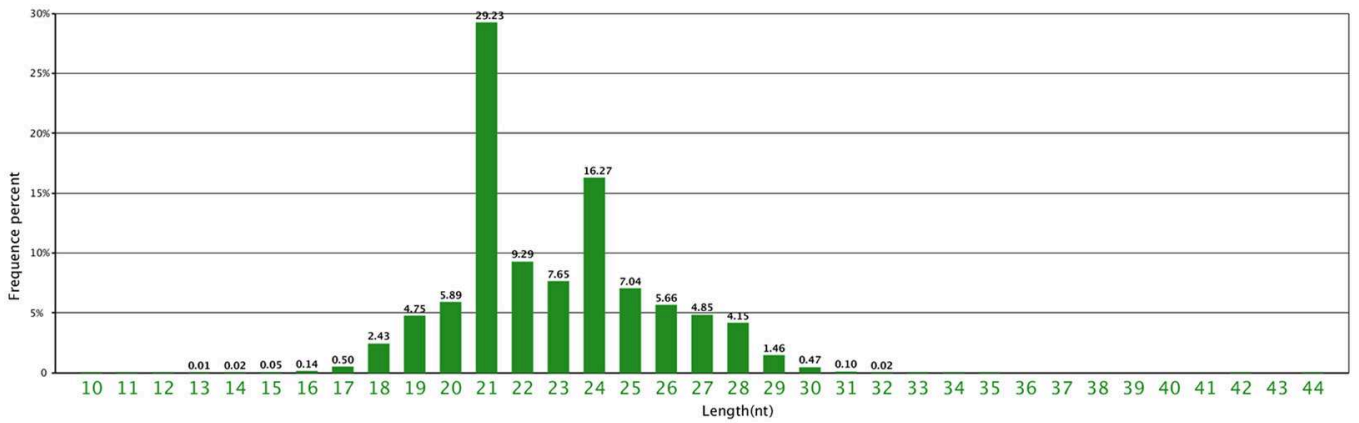

B

Length Distribution

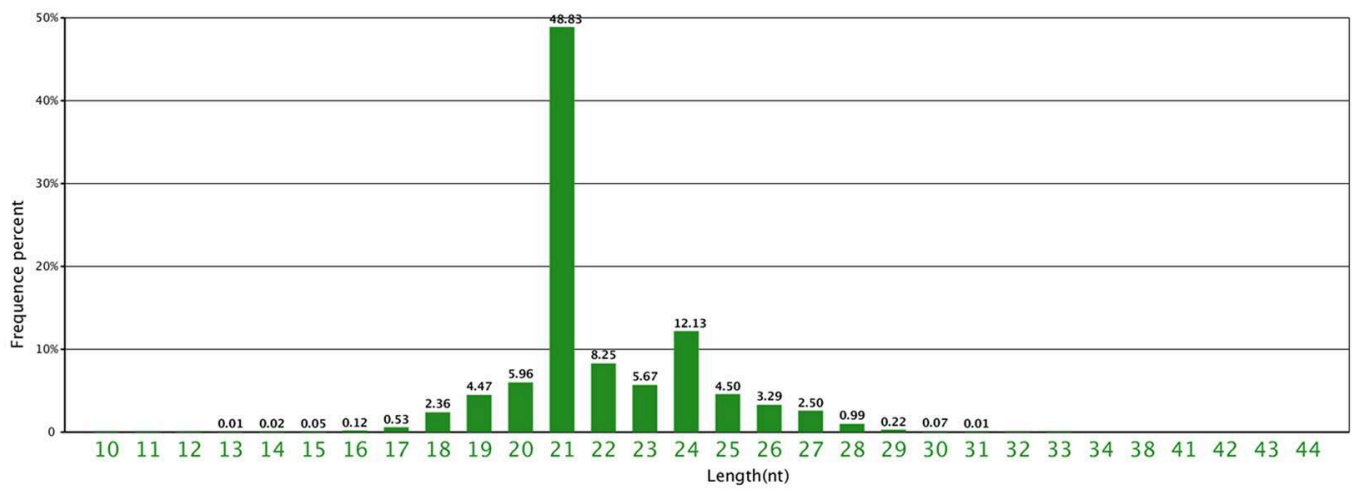

FIGURE 2 | Small RNA length distribution. Length distribution of small RNAs in (A) CA and (B) NA.

TABLE 1 | Statistic of small RNA sequences.

\begin{tabular}{|c|c|c|c|c|}
\hline & \multicolumn{2}{|c|}{ Unique sRNAs } & \multicolumn{2}{|c|}{ Total sRNAs } \\
\hline rRNA & $101,671(4.87 \%)$ & 92, $394(3.14 \%)$ & 9, 493, 261 (48.68\%) & $5,313,742(27.66 \%)$ \\
\hline snRNA & $2327(0.11 \%)$ & $1995(0.07 \%)$ & $18,534(0.10 \%)$ & $8704(0.05 \%)$ \\
\hline snoRNA & $848(0.04 \%)$ & $955(0.03 \%)$ & $3716(0.02 \%)$ & $3410(0.02 \%)$ \\
\hline Unann & 1, 955, 441 (93.69\%) & $2,820,464(95.72 \%)$ & $8,811,528(45.19 \%)$ & $12,415,657(64.62 \%)$ \\
\hline Total & $2,087,133(100 \%)$ & 2, 946, $621(100 \%)$ & 19, 500, $319(100 \%)$ & $19,212,618(100 \%)$ \\
\hline
\end{tabular}




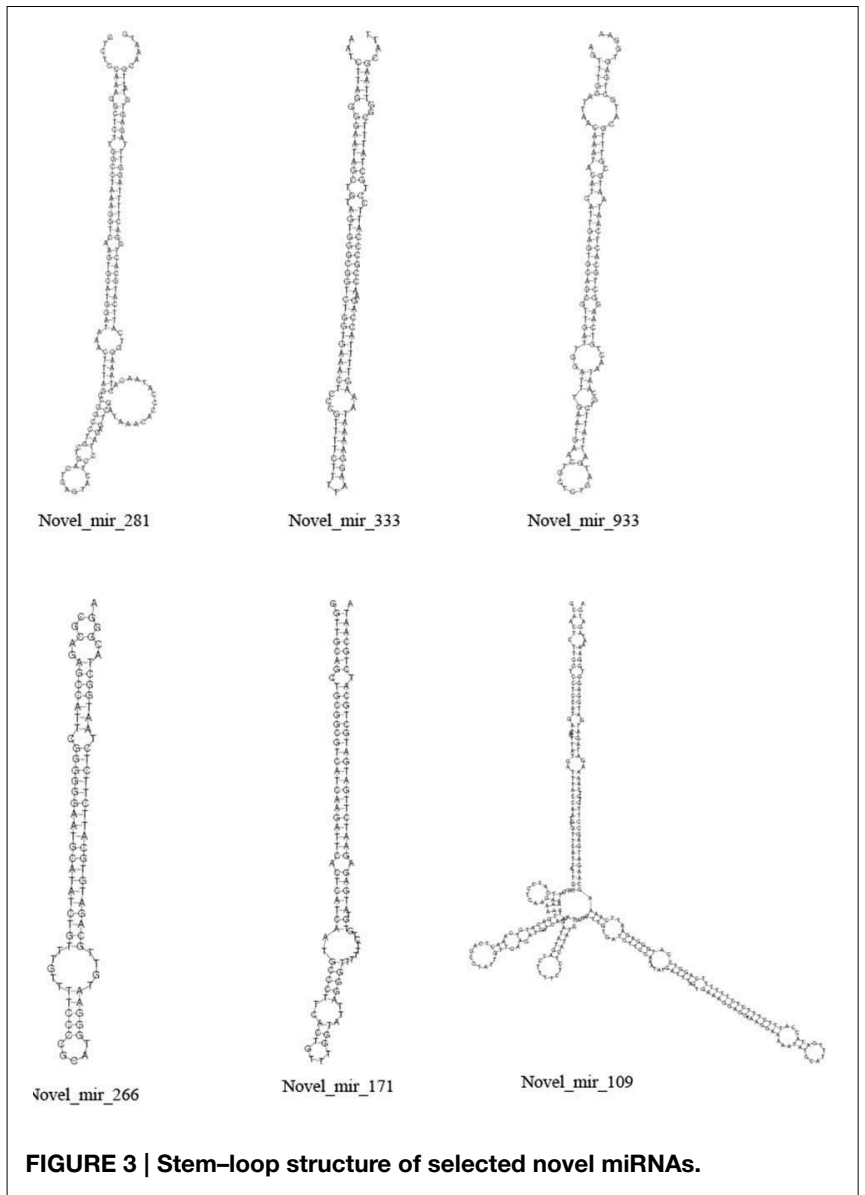

novel_mir_171 became the highest in NA, although all three were hardly detected in CA (Table S2). Quantitative real-time PCR (qRT-PCR) was conducted, and the results agreed with the high-throughput dataset (Figure S1).

Among the 336 known miRNAs, 265 (78.87\%) were differentially expressed. Only 178 miRNAs (approximately $67.17 \%$ of the 265 DE-miRNAs) were upregulated in NA. In addition, 221 miRNAs ( $79.78 \%$ of the 277 novel miRNAs) were differentially expressed, whereas 134 (60.63\%) were upregulated in NA (Table S2). In summary, known and novel miRNAs exhibited highly diverse expression patterns, thereby indicating their important roles during long-term subculture.

\section{Prediction and Annotation of DE-miRNA Targets}

To understand the possible biological functions of DE-miRNAs, mRNA-seq was simultaneously performed. Among the 265 known and 221 novel DE-miRNAs, 150 known and 137 novel miRNAs targeted 481 and 449 genes, respectively, with a total of 914 target genes (Table S3). The cleavage information is shown in Table S4. GO (Gene Ontology) enrichment analysis showed that the targets were mostly enriched in "cellular process," "metabolic process," "cell part," "binding," and "catalytic activity" (Figure 5). Further GO enrichment multiple analysis showed that "energy coupled proton transmembrane transport, against electrochemical gradient” (GO: 001598814),
"ATP hydrolysis coupled proton transport" (GO: 0015991), "baruol synthase activity" (GO: 0080011), "thioglucosidase activity" (GO: 0019137), and "transcription factor TFIIE complex" (GO: 0005673) were the enriched GO terms, thereby indicating that known DE-miRNAs regulated these processes during long-term subculture. Meanwhile, "detoxification of nitrogen compound" (GO: 0051410), "response to nitrosative stress" (GO: 0051409), "response to L-ascorbic acid" (GO: 0033591), "cyanoalanine nitrilase activity" (GO: 0047427), "indole-3-acetonitrile nitrile hydratase activity" (GO: 0080109), "indole-3-acetonitrile nitrilase activity" (GO: 0080061), and "3-cyanoalanine hydratase activity" (GO: 0047558) were the most enriched terms of novel DE-miRNAs (Figure S2).

The up- and down- regulated targets were separately subjected to GO analysis. According to our results, several pathways were increased in CA but were not found in NA, such as the pathways for antioxidants, electron carriers, biological adhesion, and ribonucleoprotein complexes. Although the genes of these pathways account for a small percentage of the DE targets, their presence indicated that CA was more likely to have a better lifespan (Figure S3).

Only 282 and 218 genes with KEGG pathway annotation were targeted by known and novel miRNAs, respectively. Among the targets of known miRNAs, "metabolic pathways," "biosynthesis of secondary metabolites," "plant-pathogen interaction," "plant hormone signal transduction," "pyrimidine metabolism," "purine metabolism," "RNA transport," and "oxidative phosphorylation" were the highly ranked pathways (Table S5). Among the targets of novel miRNAs, "metabolic pathways," "biosynthesis of secondary metabolites," and "protein processing in endoplasmic reticulum" were the most highly ranked. These results indicated that these pathways were the main targets of DE-miRNAs during long-term subculture.

\section{Analysis of DE-miRNA and their Target Genes}

Among the 150 known DE-miRNAs with target genes, 110 were upregulated and 40 were downregulated. Among their 481 target genes, 245 genes (50.94\%) were differentially expressed in NA, 117 were upregulated, and 127 were downregulated. The 110 upregulated known miRNAs targeted 390 genes, with 98 upregulated and 93 downregulated. The 40 downregulated known miRNAs targeted 104 genes, with 24 upregulated and 41 downregulated (Table S3).

Moreover, among the 137 novel miRNAs, 83 were upregulated and 54 were downregulated. For the 449 target genes, 256 (57.02\%) were differentially expressed genes in NA, in which 108 were upregulated and 148 were downregulated. The 83 upregulated novel miRNAs targeted 293 genes, with 76 upregulated and 91 downregulated. The 54 downregulated novel miRNAs targeted 181 genes, with 38 upregulated and 63 downregulated.

\section{miRNA/mRNA Modules Mainly Involved in Primary Metabolism and Abiotic or Biotic Stress Responses Oxidative Phosphorylation}

A total of 11 downregulated genes among the 22 target genes were targeted by 8 known and 2 novel upregulated miRNAs. All 


\section{A}

Scatter plot (control:x | treatment:y)

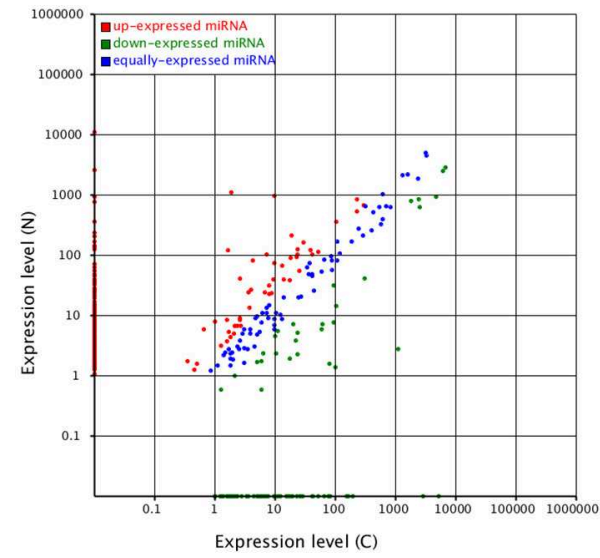

B Scatter plot (control:x | treatment:y)

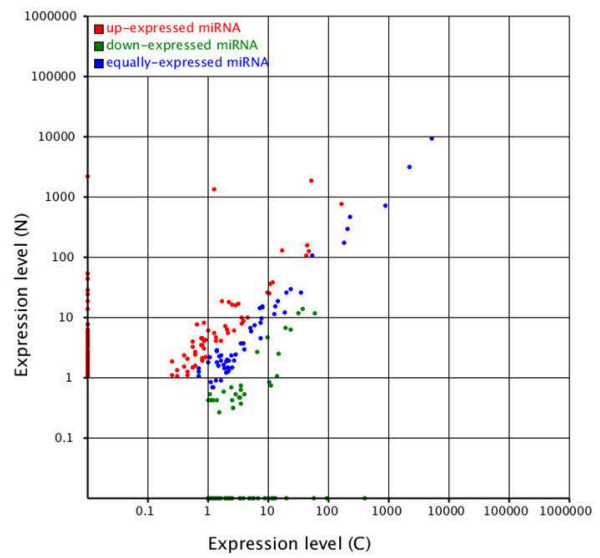

FIGURE 4 | Expression levels of miRNAs. Expression levels of (A) known miRNAs and (B) novel miRNAs.

miRNAs were barely expressed in CA, thereby indicating that oxidative phosphorylation was mainly regulated by miRNAs. The 11 downregulated genes included cytochrome $c$ oxidase subunit 2, succinate dehydrogenase (ubiquinone) cytochrome b560 subunit, V-type $\mathrm{H}+$-transporting ATPase subunit F, NAD(P)Hquinone oxidoreductase subunit 5, and $\mathrm{NADH}$-ubiquinone oxidoreductase chains 2 and 4 (Table S6).

\section{RNA Polymerase}

Only 13 of the 21 targeted genes were targeted by $15 \mathrm{DE}$ known miRNAs (13 upregulated and 4 downregulated) and 2 upregulated novel miRNAs. Among these genes, 9 targets were differentially expressed, in which 5 were upregulated and 4 were downregulated. Ten modules were inversely related, with 4 upregulated miRNAs, namely, miR5056, miR5499, miR6230-3p, and miR7719, which downregulated two genes that coded for the DNA-directed RNA-polymerase subunit $\beta$. The downregulated miR5509 and miR7812 were inversely related to the RPABC subunit of RNA polymerases I, II, and III, as well as the maintenance of ploidy protein MOB1 (MPS1 binder 1) (Table S6).

\section{Pyrimidine and Purine Metabolism}

A total of 25 targeted genes were targeted by 22 DE known miRNAs (17 upregulated and 5 downregulated) and 7 novel miRNAs (6 upregulated and 1 downregulated). Only 15 targets were differentially expressed, in which 8 were upregulated and 7 were downregulated. Likewise, 15 modules were inversely related. For example, DNA-directed RNA polymerase, DNA polymerase, and ATP-dependent DNA helicase RecG were downregulated by the respective miRNAs (Table $\mathbf{S 6}$ ).

Additionally, several inversely related miRNA/mRNA modules were involved in RNA transport, DNA replication, ribosomes, and so on. These results indicated that miRNAs had important roles in regulating bioactivity for the supply of essential materials and energy, such as oxidative phosphorylation and RNA polymerase. Moreover, most of the miRNAs were upregulated, whereas their targets were downregulated in NA, thereby indicating that these pathways were repressed by miRNAs during long-term subculture.

\section{Plant Hormone Signal Transduction and Plant-pathogen Interaction}

A total of 52 genes were targeted by 30 DE-miRNAs (24 upregulated and 6 downregulated). Only 30 targets were differentially expressed, with 18 upregulated and 12 downregulated. Among the 13 inversely related miRNA/mRNA modules, brassinosteroid insensitive 1-associated receptor kinase (miR5819), disease resistance protein RPS2 (miR6161d), mitogen-activated protein kinase kinase (miR7535), mitogenactivated protein kinase kinase kinase (miR2919), auxin response factor (miR7540a), pathogenesis-related protein 1 (novel_mir_109), and extracellular signal-regulated kinase (miR1044-3p) were downregulated. By contrast, transcription factor TGA (novel_mir_73) and disease resistance protein RPM1 (miR821a) were upregulated. These results indicated that the $\mathrm{SA}$, brassinosteroid, and auxin signal transduction processes, as well as the plant-pathogen interaction responses, were mainly regulated by miRNAs during long-term subculture. Additionally, CA seemed to be more sensitive to abiotic or biotic stress signals (Table S6).

\section{miRNA/mRNA Modules are Involved in Phenylpropanoid, Flavonoid, and Terpenoid Biosynthesis}

Phenylpropanoid, flavonoids, and terpenoid are the most dominant secondary metabolites in plants. Their miRNA regulatory mechanisms are representative models for studying secondary metabolism during long-term subculture.

Among the 32 targeted genes, 19 were targeted by 10 upregulated known miRNAs and 1 downregulated novel miRNA. A total of 12 targets were differentially expressed, in which 11 were upregulated and 1 was downregulated. Among the $14 \mathrm{miRNA} / \mathrm{mRNA}$ modules, only 2 were inversely 


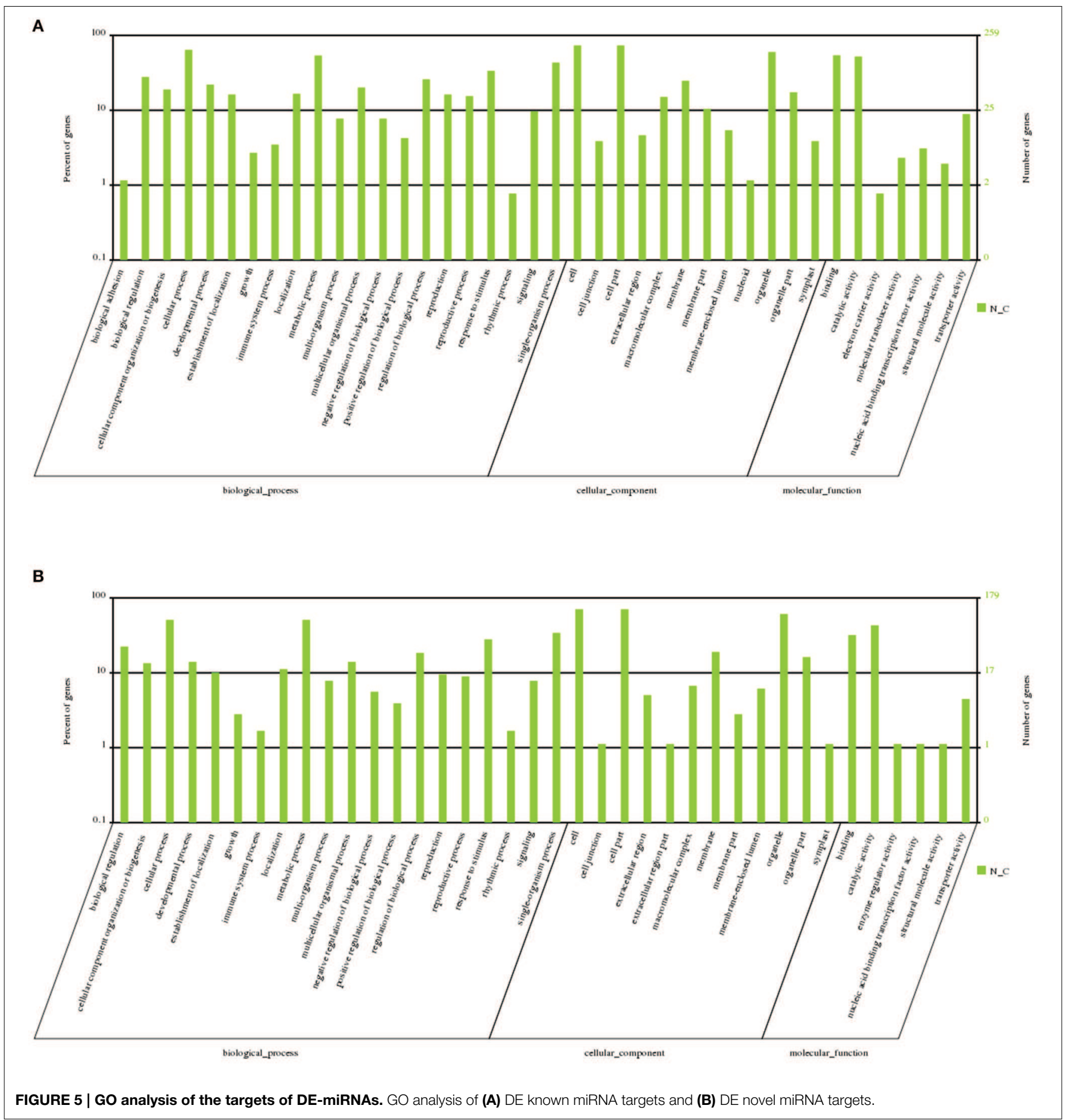

related: miR7696a-5p/peroxidase and novel_mir_331/gibberellin 2-oxidase.

The taxol biosynthesis genes TAT, T5H, and $\mathrm{T} 10 \mathrm{H}$ were targeted by miRNAs. miR2645, which targets TAT, was

upregulated. However, its expression was not inversely related. miRNAs seem to have a limited role in the regulation of secondary metabolism.
miR8154 and miR5298b are Important Regulators of Long-term Subculture

Overall, 1059 miRNA/mRNA modules were identified, but only 275 (25.97\%) were inversely related. Additionally, two known upregulated miRNAs, miR8154 and miR5298b, were identified as important regulators in the network by the Cytoscape software (download available at http://www.cytoscape.org/). 
These miRNAs regulated a series of genes involved in various pathways, including transcription factors, methyltransferases, and functional enzyme genes (Table S7; Figure 6). Both miRNAs were inversely related to $11 \mathrm{DE}$ target genes during long-term subculture, including histone arginine demethylase, presenilin enhancer 2, ribosomal protein S21, and translation initiation factor 3. These results indicated that the methylation level, protein processing, and transcriptional level changed during long-term subculture, and these miRNAs and genes may be correlated to the conversion of subculture.

miR8154 and miR5298b were over-expressed in CA, and the expression of the five selected genes was quantitatively detected in Taxus cells. WRKY33 and miR5298b followed an updown module according to our dataset, whereas WRKY33 was downregulated by 3.99 times in the miR5298b-overexpressing lines. These results confirmed that miR8154/miR5298b regulated their targets as revealed by the high-throughput dataset. To detect whether the two miRNAs affected the secondary metabolism, the expression of tasy (taxadiene synthase), dbat (10deacetylbaccatin III-10-O-acetyl transferase), pal (phenylalanine ammonia lyase), and chs, which are the respective key genes of taxol, phenylpropane, and flavonoid biosynthesis, were detected. Interestingly, all these genes were significantly upregulated in miR8154- and miR5298b-overexpressing cells (Figure 7). However, miR8154 and miR5298b could not target these genes. According to our high-throughput dataset, miR8154 and miR5298b were upregulated in NA cells. Their overexpression seemed to promote the recovery of metabolic networks in CA to their initial status, which was similar to that in NA. These results indicated that miR8154 and miR5298b had important roles in the network conversion during long-term subculture.

\section{Discussion}

Subcultured cells were separated from cooperated organisms, with a series of significant differences after long-term subculture. For example, our work showed that long-term subcultured cells turned white from brown, and their production greatly changed in terms of composition and yield. Unlike natural bioactivity, the conversion of long-term subcultured cells was a result of multiple cellular bioactivity involving comprehensive metabolic pathways. However, previous reports focused on epigenetic variation, morphological stability, gene mutations, and so on; none of these reports studied the altered expression and regulatory systems of the long-term subcultured cells (Kiselev et al., 2011; Miguel and Marum, 2011; Smulders and De Klerk, 2011; Neelakandan and Wang, 2012; Wang and Wang, 2012; Rival et al., 2013; Kwiatkowska et al., 2014). Differences in the gene expression profiles could prominently reveal the responses of cells during adaptation to novel environments. miRNAs are known to control processes, such as development, signal transduction, response to environmental stress, and pathogen invasion, mainly at the posttranscriptional level (He and Hannon, 2004; Mahajan et al., 2011; Sun, 2012; Sunkar et al., 2012; Zeng et al., 2014). Thus, miRNA regulation has a crucial role. Consequently, two Taxus cell lines, CA (subcultured for 10 years) and NA (freshly separated and subcultured for 6 months), were quantified at the mRNA and miRNA levels by high-throughput sequencing in this study. Our

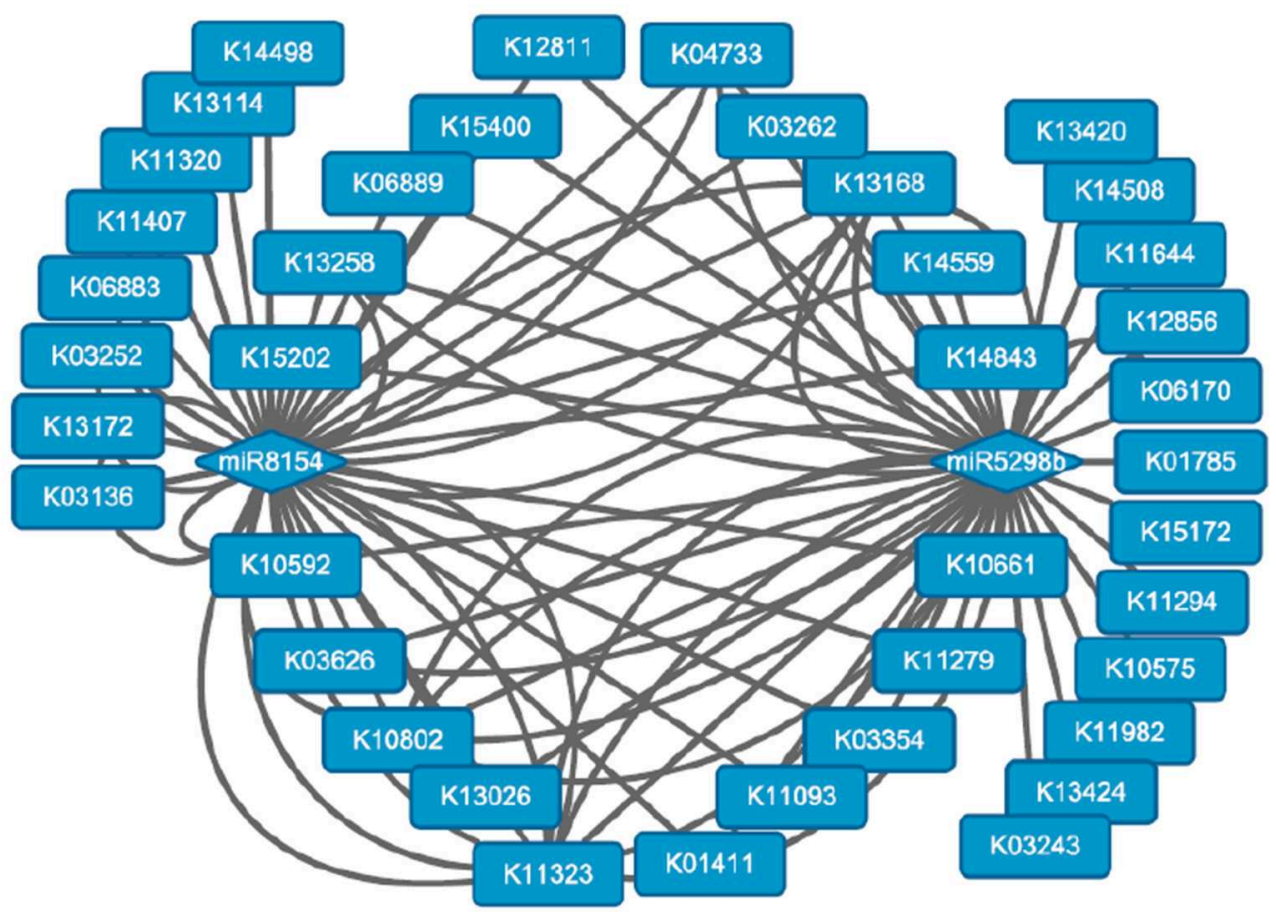

FIGURE 6 | Biological pathways regulated by miR8154 and miR5298b. The round rectangles represented the KO IDs of the KEGG pathways. 


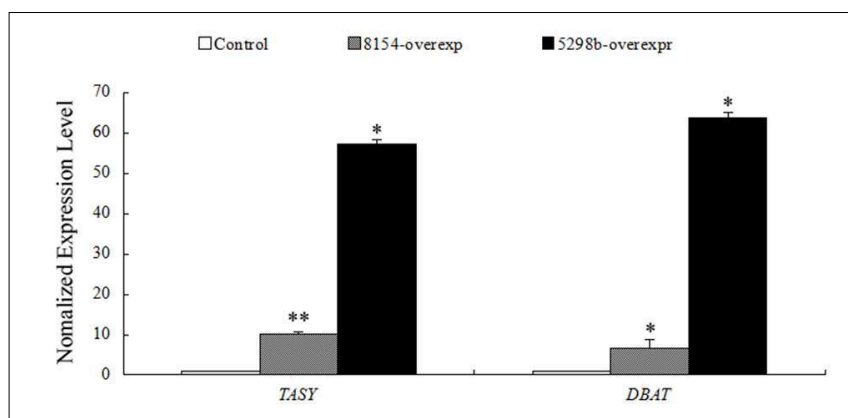

FIGURE 7 | Expression profiles of selected genes in CA cells with miR8154 and miR5298b overexpression. Tasy, Taxadiene synthase; DBAT, 10-deacetylbaccatin III-10-O-acetyl

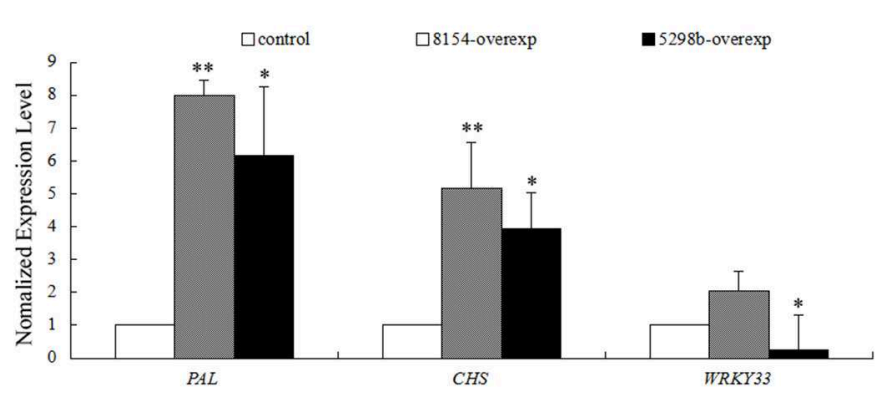

transferaseferase; PAL, phenylalanine ammonia lyase; $\mathrm{CHS}$, chalcone synthase. Error bars indicate SE. ${ }^{* *}$ indicates $p<0.01$; *indicates $p<0.05$. data could promote the understanding of miRNA regulatory systems in plants and give insights into the long-term subculture of cells.

Plant miRNAs only have a small number of mRNA targets $(\sim 150)$, which is less than $1 \%$ of the protein-coding genes in Arabidopsis (Addo-Quaye et al., 2008; German et al., 2008; Li et al., 2010; Sunkar et al., 2012). Our data showed that 915 genes were targeted by known and novel miRNAs, which was approximately $2 \%$ of the coding genes; known miRNAs targeted 481 protein-coding genes (approximately 1\%). These results indicated that our miRNA data had excellent coverage. Among these targets, three taxol biosynthetic enzyme genes (TAT, T10H, and $\mathrm{T} 5 \mathrm{H}$ ) were identified as miRNA targets for the first time in this study. This information provides a foundation for studying the miRNA-directed regulation of taxol biosynthesis (Qiu et al., 2009; Hao et al., 2012). Several genes were also newly targeted by miRNAs, especially by some novel miRNAs. These data could improve the current understanding of miRNA regulation.

The miRNAs regulate their targets through at least four mechanisms. Translational inhibition of the targets is the most important regulatory mechanism in animals, whereas direct cleavage of the target mRNAs is predominant in plants (Chen, 2009; Jones-Rhoades, 2012; Pei et al., 2013). Therefore, the expression of most plant miRNAs and their targets are inversely related. Several reports of miRNA regulatory systems focused on the inversely related miRNA/mRNA modules (Ori et al., 2007; Yang et al., 2012; Zeng et al., 2014). Among the 1058 DE-miRNA/mRNA modules in our dataset, only 275 modules (26.0\%) were inversely related, whereas another 302 modules (28.5\%) were positively related. The mRNAs of 481 modules (45.5\%) had no significant differential expression. According to our data, the positively related modules had an equal number as the inversely correlated miRNA/target pairs. These results were highly similar to the work of Lopez-Gomollon et al. (2012). In addition, previous reports indicated that noninversely related modules were functional groups (Kidner and Martienssen, 2004; Nikovics et al., 2006; Kawashima et al., 2009), but our study focused mainly on the inversely related modules.

Among the 275 inversely related modules, 68 miRNAs (39 known and 29 novel) and 114 potential targets formed 125 modules with functional annotation. A total of 101 modules $(80.8 \%)$ were up-down modules, which indicated that the genes were repressed in NA, including those for the ATPbinding cassette, transcription initiation factor TFIID, E3 ubiquitin-protein ligase synoviolin, ATP-dependent DNA helicase, protein transport protein, $5^{\prime}-3^{\prime}$ exoribonuclease, DNA mismatch repair protein, and histone arginine demethylase, among others. Moreover, most genes of the affected pathways, including oxidative phosphorylation, RNA polymerase, purine and pyrimidine metabolism, plant hormone signal transduction, and plant-pathogen interaction, were also downregulated in NA. All these results indicated that miRNAs were one of the essential regulators of primary metabolism during long-term subculture. Plant hormone signal transduction and plant-pathogen interaction are important pathways in plant defense, development, and stress responses; our results indicated that they were also mainly regulated by miRNAs.

According to the above results, several somatic mutations were related to miRNAs. First, the long-term subcultured cells grew faster than the newly derived cells because of the stronger primary metabolism. For example, stronger activity of oxidative phosphorylation could provide more energy for cell growth. Activities such as oxidative phosphorylation and DNA replication were mainly regulated by several differentially expressed miRNAs. Second, long-term subcultured cells were more likely to undergo gene mutation. In CA, several components of the mismatch recognition complex were not upregulated with the increased DNA replication. The expression of MSH6, PMS2 (downregulated), and MLH1 (unDE) indicated that the possibility of errors was higher when DNA is replicated. The functions of the miR6167/MSH6 (both upregulated in CA) and novel_mir_327/MLH1 (downunDE modules) modules should be further clarified for gene mutations. Moreover, the targets that were annotated as multiorganism processes by GO analysis were all downregulated in CA. Therefore, these miRNAs, including miR5509, miR1312, and miR7812, among others, were related to cellular morphological changes (Table S3). Additionally, miRNAs were inversely related to several genes that encoded methylases and demethylases, thereby indicating 
that miRNAs probably affected the epigenetic modifications (Table S3).

By contrast, the biosynthesis of phenylpropanoid, flavonoids, and terpenoid was rarely affected by miRNA. Among the 18 modules formed by 11 miRNAs and 16 genes, which were related to these pathways, only the miR7696a-5p/peroxidase and novel_mir_331/gibberellin 2-oxidase modules were inversely related. All other relevant miRNAs were upregulated, and most of their targets were upregulated in NA, except for cinnamyl alcohol dehydrogenase, UDP-glucosyl transferase, gibberellin 3 - $\beta$-dioxygenase, and naringenin 3 -dioxygenase. The four genes were not significantly differentially expressed during long-term subculture. These results indicated that although miRNAs could regulate secondary metabolism, differences in the secondary metabolism were not directly affected by miRNAs during long-term subculture. Perhaps miRNAs functioned in an indirect manner to influence secondary metabolites. According to Alexandre et al. miR393 could repress auxin signaling to prevent auxin signaling from antagonizing SA signaling. Thus, miR393 re-directs secondary metabolite biosynthesis away from camalexin and toward glucosinolates (Robert-Seilaniantz et al., 2011). In our data, plant hormone signal transduction and plant-pathogen interaction were apparently regulated by miRNAs; even miR7540a could suppress auxin response factors such as miR393 in NA. Therefore, miRNAs may indirectly redirect secondary metabolite biosynthesis. Additionally, the upregulated miR8154 and miR5298b were identified to control a series of genes involved in various pathways, including those encoding transcription factors, methyltransferases, and functional enzymes. Their overexpression showed that the key genes of secondary metabolite biosynthesis were significantly upregulated, although these genes were not targeted by miR8154 and miR5298b. Interestingly, overexpression of solely miRNA was not capable to increase the secondary metabolites significantly, indicating the two miRNA were not the most original factors of the conversion. The conversion of subcultured cells were considered as the integrative results of many factors including miRNAs. Further analysis may help us understand the complex regulatory network of long-term subculture.

Overall, the secondary metabolite production of long-term subcultured cells decreased. Variations, such as epigenetic variation, morphological stability, gene mutations, and cellular ploidy levels, were considered to be the partial reason for the decreased production. Moreover, our work showed that long-term subcultured cells converted their production properties, thereby increasing primary metabolism and decreasing secondary metabolism. Given the limited production capacity of cells, conversion should be beneficial for cell growth. Therefore, conversion seems to be a positive response to novel environments, and factors such as epigenetic variation, morphological stability, gene mutations, and cellular ploidy level contributed to the positive conversion. Our results indicated that miRNAs are comprehensively involved in the conversion during long-term subculture. The primary metabolism and secondary metabolism are targets of the miRNA regulatory system. In particular, primary metabolism was effectively and principally regulated by miRNAs. In addition, secondary metabolism was not regulated by the miRNA regulatory system. The effects of the miRNA regulatory system on secondary metabolism were very faint during long-term subculture. For example, long-term subcultured cells increased their rRNA levels by 1.8 times. Interestingly, a large number of unknown $21 \mathrm{nt}$-length sRNAs was simultaneously reduced, thereby indicating that the unknown 21 nt-length sRNAs had important roles during conversion. No tissue is immune to free viral infections in plants and retroviral elements can be from the plant genome although the callus were detoxified. Therefore, considering the inserted virus genome in plants, virus-derived siRNAs may be a reason of the accumulation of unknown sRNAs in NA. Further studies about the unannotated 21 nt-length sRNAs should be conducted to clarify the novel regulation factor of the conversion during subculture. The discovery of miR8154, miR5298b, taxol biosynthetic genes, and other functional genes provides abundant information on the miRNA regulatory system and could promote our understanding of the long-term subculture of plant cells.

\section{Materials and Methods}

\section{Plant Materials}

CA was established from callus cultures derived from cultureinitiated embryos, which were excised from the nascent tender stems of Taxus chinensis in May 2003, the callus were detoxified, and maintained with modified Gamborg's B5 medium at $25^{\circ} \mathrm{C}$ in the dark (Li et al., 2012). The NA cell line was established from callus cultures, which were newly derived from $T$. chinensis leaves in May 2013 and maintained with modified Gamborg's B5 medium for 6 months before RNA extraction. In addition, $\mathrm{CA}$ and NA were derived from the same organism, which was cultivated in the nursery of the Huazhong University of Science and Technology.

\section{Total RNA Extraction}

Cells were harvested in a microfuge tube, immersed in TRIzol Regent (Invitrogen, USA), and immediately frozen in liquid nitrogen before total RNA extraction. The E.Z.N.A. ${ }^{\circledR}$ Plant RNA Kit (Omega, USA) can effectively inhibit the RNA degradation influenced by polysaccharides and phenolic compounds in NA cells; thus, the kit was used to extract the total RNA from all samples. Total RNA of each cell line was treated with DNase I (Invitrogen) and sent to Beijing Genomics InstituteShenzhen (Shenzhen, China) for mRNA/miRNA purification and sequencing with Illumina HiSeq2000 technology. Samples of NA and CA were mixed with RNA from three independent bio-repeats. Moreover, two other samples were sent for highthroughout sequencing to validate the results of NA and CA.

\section{Assembly and Analysis mRNA-seq}

The assembly analysis of mRNA-seq was conducted according to our previous report (Li et al., 2012).

\section{miRNA-seq}

The 18-30 nt long small RNAs were collected. The $5^{\prime}$ and $3^{\prime}$ adaptors were added before sequencing by synthesis (SBS) 
with Hiseq2000 technology. The 49 nt long sequence tags were subjected to data cleaning analysis to obtain credible clean tags. The Tag2miRNA software annotated these clean tags into different categories via alignment with miRBase by taking full account of miRNA conservation. First, two mismatches and free gaps were allowed when aligning clean tags to the miRNA precursor/mature miRNA of all plants/animals in miRBase by considering the differences among species. Second, the miRNA with the highest expression was chosen for each mature miRNA family and added to a temporary miRNA database. All the annotated tags were designated as known miRNAs. For the nonannotated tags, the Mireap software was used to predict the novel miRNAs by alignment with the simplified T. baccata genome; the algorithm was set according to a previous report (Zhang et al., 2013). The miRNAs that could not fold into hairpin structures were regarded as pseudo-miRNAs (Nystedt et al., 2013). The known and novel miRNAs were aligned with the simplified $T$. baccata genome to verify their hairpin structure. The known miRNAs and novel miRNAs of the two samples were obtained. Subsequently, the targets of the miRNAs were predicted (Allen et al., 2005; Schwab et al., 2005), whereas GO enrichment and KEGG pathway analysis were performed on the target genes.

\section{Identification of Differentially Expressed Tags miRNA-seq}

The expression of known miRNAs was generated by adding the number of tags that aligned to the temporary miRNA database within two mismatches. The expression of novel miRNA is produced by adding the number of miRNAs with no more than three mismatches on the $5^{\prime}$ and $3^{\prime}$ ends but with no mismatches in the middle based on the alignment result. The same method was used to identify differentially expressed miRNA for the known and novel miRNAs. First, the expression of miRNA in both samples (CA and NA) was normalized to obtain the expression in TPM: TPM = actual miRNA count/total count of clean reads $\times 1,000,000$. Second, the fold-change was calculated and represented as log 2(TPM-NA/TPM-CA). The $P$-value of TPM for the two samples was also determined. miRNAs with a fold-change of $>1$ or $<-1$ and $p<0.05($ FDR $\leq 0.05)$ were considered to be differentially expressed and designated as DEmR. The DEmRs with $p<0.01$ were considered to have significant differential expression.

\section{Vector Construction and Transient Transformation}

First, the mature miRNA and miRNA* sequences of premiR319 were replaced by the mature miR8154 and miR5298b sequences, respectively, which constituted the artificial premiR8154 and pre-miR5298b. The artificial sequences were cloned into pBI121 to replace the GUS gene and construct the respective overexpression vectors. The transient transformation was conducted as described in a previous report (Li et al., 2012).
Changes in the expression of both miRNAs were verified by qRT-PCR in transient cells.

\section{qRT-PCR}

For the qRT-PCR of mRNAs, $1 \mathrm{mg}$ of the DNase I-treated total RNA was used to synthesize cDNA with M-MLV (Promega) and poly $(\mathrm{dT})_{18}$ oligonucleotides. The U6 snRNA was obtained by a BLAST search of the T. baccata genome sequences with the U6 sequence in Arabidopsis thaliana; this snRNA was used as the reference gene (Nystedt et al., 2013). SYBR Green PCR Master Mix (Applied Biosystems) was used in all qRT-PCR experiments. Changes in the relative expression of miRNAs and genes were calculated via the $2 \Delta \Delta \mathrm{C}_{\mathrm{t}}$ method. Primers used in all the qRTPCR experiments are listed in Table S8.

\section{Author Contributions}

Conceived and designed the experiments: CF, MZ, and LY. Performed the experiments: YD, MZ, and LN. Analyzed the data: $\mathrm{MZ}$ and CF. Contributed reagents/materials/analysis tools: LY, CF, ML. Wrote the paper: MZ, CF, and LY.

\section{Acknowledgments}

This work was supported by the National Natural Science Foundation of China (31270342 and 20906036) and Science and Technology Support Program of China (Grant No.2008BAI63B04).

\section{Supplementary Material}

The Supplementary Material for this article can be found online at: http://journal.frontiersin.org/article/10.3389/fpls.2015. 00604

Figure S1 | qRT-PCR of several selected miRNAs.

Figure S2 | Enrichment analysis of miRNA targets.

Figure S3 | GO analysis of up- and downregulated targets.

Table S1 | Quality statistics of sequencing tags of miRNAs.

Table S2 | Expression profiles of miRNAs.

Table S3 | Targets of miRNAs.

Table S4 | Target and cleavage sites of miRNAs.

Table S5 | KEGG analysis of miRNA targets.

Table S6 | miRNA/target modules involved in several pathways.

Table S7 | Targets of miR8154 and miR5298b.

Table S8 | Primers used in qRT-PCR. 


\section{References}

Addo-Quaye, C., Eshoo, T. W., Bartel, D. P., and Axtell, M. J. (2008). Endogenous siRNA and miRNA targets identified by sequencing of the Arabidopsis degradome. Curr. Biol. 18, 758-762. doi: 10.1016/j.cub.2008.04.042

Allen, E., Xie, Z., Gustafson, A. M., and Carrington, J. C. (2005). microRNAdirected phasing during trans-acting siRNA biogenesis in plants. Cell 121, 207-221. doi: 10.1016/j.cell.2005.04.004

Baebler, S., Hren, M., Camloh, M., Ravnikar, M., Bohanec, B., Plaper, I., et al. (2005). Establishment of cell suspension cultures of yew (Taxus x Media Rehd.) and assessment of their genomic stability. In Vitro Cell. Dev. Biol. Plant 41, 338-343. doi: 10.1079/IVP2005642

Bartel, D. P. (2009). MicroRNAs: target recognition and regulatory functions. Cell 136, 215-233. doi: 10.1016/j.cell.2009.01.002

Chen, F., Zhang, W., Liang, Y., Huang, J. L., Li, K., Green, C. D., et al. (2012). Transcriptome and network changes in climbers at extreme altitudes. PLoS ONE 7:e31645. doi: 10.1371/journal.pone.0031645

Chen, X. (2009). Small RNAs and Their Roles in Plant Development. Annu. Rev. Cell Dev. Biol. 25, 21-44. doi: 10.1146/annurev.cellbio.042308.113417

German, M. A., Pillay, M., Jeong, D. H., Hetawal, A., Luo, S., Janardhanan, P., et al. (2008). Global identification of microRNA-target RNA pairs by parallel analysis of RNA ends. Nat. Biotechnol. 26, 941-946. doi: 10.1038/nbt1417

Hall, R. D., and Yeoman, M. M. (1987). Intercellular and intercultural heterogeneity in secondary metabolite accumulation in cultures of Catharanthus roseus following cell line selection. J. Exp. Bot. 38, 1391-1398. doi: 10.1093/jxb/38.8.1391

Hao, D. C., Yang, L., Xiao, P. G., and Liu, M. (2012). Identification of Taxus microRNAs and their targets with high-throughput sequencing and degradome analysis. Physiol. Plant. 146, 388-403. doi: 10.1111/j.1399-3054.2012.01668.x

He, C. Y., Cui, K., Zhang, J. G., Duan, A. G., and Zeng, Y. F. (2013). Nextgeneration sequencing-based mRNA and microRNA expression profiling analysis revealed pathways involved in the rapid growth of developing culms in Moso bamboo. BMC Plant Biol. 13:119. doi: 10.1186/1471-2229-13-119

He, L., and Hannon, G. J. (2004). MicroRNAs: small RNAs with a big role in gene regulation. Nat. Rev. Genet. 5, 522-531. doi: 10.1038/nrg1379

Howat, S., Park, B., Oh, I. S., Jin, Y. W., Lee, E. K., and Loake, G. J. (2014). Paclitaxel: biosynthesis, production and future prospects. N. Biotechnol. 31, 242-245. doi: 10.1016/j.nbt.2014.02.010

Jones-Rhoades, M. W. (2012). Conservation and divergence in plant microRNAs. Plant Mol. Biol. 80, 3-16. doi: 10.1007/s11103-011-9829-2

Jones-Rhoades, M. W., and Bartel, D. P. (2004). Computational identification of plant MicroRNAs and their targets, including a stress-induced miRNA. Mol. Cell 14, 787-799. doi: 10.1016/j.molcel.2004.05.027

Kawashima, C. G., Yoshimoto, N., Maruyama-Nakashita, A., Tsuchiya, Y. N., Saito, K., Takahashi, H., et al. (2009). Sulphur starvation induces the expression of microRNA-395 and one of its target genes but in different cell types. Plant J. 57, 313-321. doi: 10.1111/j.1365-313X.2008.03690.x

Kidner, C. A., and Martienssen, R. A. (2004). Spatially restricted microRNA directs leaf polarity through ARGONAUTE1. Nature 428, 81-84. doi: 10.1038/nature02366

Kiselev, K. V., Shumakova, O. A., and Tchernoded, G. K. (2011). Mutation of Panax ginseng genes during long-term cultivation of ginseng cell cultures. J. Plant Physiol. 168, 1280-1285. doi: 10.1016/j.jplph.2011.01.028

Kolewe, M. E., Gaurav, V., and Roberts, S. C. (2008). Pharmaceutically active natural product synthesis and supply via plant cell culture technology. Mol. Pharm. 5, 243-256. doi: 10.1021/mp7001494

Kolewe, M. E., Henson, M. A., and Roberts, S. C. (2010). Characterization of aggregate size in Taxus suspension cell culture. Plant Cell Rep. 29, 485-494. doi: $10.1007 /$ s00299-010-0837-5

Kwiatkowska, A., Zebrowski, J., Oklejewicz, B., Czarnik, J., Halibart-Puzio, J., and Wnuk, M. (2014). The age-dependent epigenetic and physiological changes in an Arabidopsis T87 cell suspension culture during long-term cultivation. Biochem. Biophys. Res. Commun. 447, 285-291. doi: 10.1016/j.bbrc.2014. 03.141

Li, L. Q., Li, X. L., Fu, C. H., Zhao, C. F., and Yu, L. J. (2013). Sustainable use of Taxus media cell cultures through minimal growth conservation and manipulation of genome methylation. Process Biochem. 48, 525-531. doi: 10.1016/j.procbio.2013.01.013
Li, S. T., Zhang, P., Zhang, M., Fu, C. H., Zhao, C. F., Dong, Y. S., et al. (2012). Transcriptional profile of Taxus chinensis cells in response to methyl jasmonate. BMC Genomics 13:295. doi: 10.1186/1471-2164-13-295

Li, Y. F., Zheng, Y., Addo-Quaye, C., Zhang, L., Saini, A., Jagadeeswaran, G., et al. (2010). Transcriptome-wide identification of microRNA targets in rice. Plant J. 62, 742-759. doi: 10.1111/j.1365-313X.2010.04187.x

Lila, M. (2005). "Valuable secondary products from in vitro culture," in Plant Development and Biotechnology, eds D. J. Gray and R. N. Trigiano (Boca Raton, FL: CRC Press), 376.

Lopez-Gomollon, S., Mohorianu, I., Szittya, G., Moulton, V., and Dalmay, T. (2012). Diverse correlation patterns between microRNAs and their targets during tomato fruit development indicates different modes of microRNA actions. Planta 236, 1875-1887. doi: 10.1007/s00425-012-1734-7

Lu, S., Sun, Y. H., Shi, R., Clark, C., Li, L., and Chiang, V. L. (2005). Novel and mechanical stress-responsive microRNAs in Populus trichocarpa that are absent from Arabidopsis. Plant Cell 17, 2186-2203. doi: 10.1105/tpc.105. 033456

Mahajan, V., Mahajan, A., Pagoch, S., Bedi, Y., and Gandhi, S. (2011). microRNA mediated regulation of plant secondary metabolism: an in silico analysis. J. Nat. Sci. Biol. Med. 2, 44-45.

Malik, S., Cusido, R. M., Mirjalili, M. H., Moyano, E., Palazon, J., and Bonfill, M. (2011). Production of the anticancer drug taxol in Taxus baccata suspension cultures: a review. Process Biochem. 46, 23-34. doi: 10.1016/j.procbio.2010.09.004

Mallory, A. C., and Vaucheret, H. (2006). Functions of microRNAs and related small RNAs in plants. Nat. Genet. 38(Suppl.), S31-S36. doi: 10.1038/ng0706$850 \mathrm{~b}$

Miguel, C., and Marum, L. (2011). An epigenetic view of plant cells cultured in vitro: somaclonal variation and beyond. J. Exp. Bot. 62, 3713-3725. doi 10.1093/jxb/err155

Mustafa, N. R., de Winter, W., Van Iren, F., and Verpoorte, R. (2011). Initiation, growth and cryopreservation of plant cell suspension cultures. Nat. Protoc. 6, 715-742. doi: 10.1038/nprot.2010.144

Naill, M. C., and Roberts, S. C. (2005). Flow cytometric identification of Paclitaxel-accumulating subpopulations. Biotechnol. Prog. 21, 978-983. doi: $10.1021 / \mathrm{bp} 0495441$

Neelakandan, A. K., and Wang, K. (2012). Recent progress in the understanding of tissue culture-induced genome level changes in plants and potential applications. Plant Cell Rep. 31, 597-620. doi: 10.1007/s00299-011-1202-z

Nikovics, K., Blein, T., Peaucelle, A., Ishida, T., Morin, H., Aida, M., et al. (2006). The balance between the MIR164A and CUC2 genes controls leaf margin serration in Arabidopsis. Plant Cell 18, 2929-2945. doi: 10.1105/tpc.106. 045617

Nystedt, B., Street, N. R., Wetterbom, A., Zuccolo, A., Lin, Y. C., Scofield, D. G., et al. (2013). The Norway spruce genome sequence and conifer genome evolution. Nature 497, 579-584. doi: 10.1038/nature12211

Ori, N., Cohen, A. R., Etzioni, A., Brand, A., Yanai, O., Shleizer, S., et al. (2007). Regulation of LANCEOLATE by miR319 is required for compound-leaf development in tomato. Nat. Genet. 39, 787-791. doi: 10.1038/ng2036

Patil, R. A., Kolewe, M. E., and Roberts, S. C. (2013). Cellular aggregation is a key parameter associated with long term variability in paclitaxel accumulation in Taxus suspension cultures. Plant Cell Tissue Organ Cult. 112, 303-310. doi: 10.1007/s11240-012-0237-3

Pei, H., Ma, N., Chen, J., Zheng, Y., Tian, J., Li, J., et al. (2013). Integrative analysis of miRNA and mRNA profiles in response to ethylene in rose petals during flower opening. PLoS ONE 8:e64290. doi: 10.1371/journal.pone.0064290

Qiu, D., Pan, X., Wilson, I. W., Li, F., Liu, M., Teng, W., et al. (2009). High throughput sequencing technology reveals that the taxoid elicitor methyl jasmonate regulates microRNA expression in Chinese yew (Taxus chinensis). Gene 436, 37-44. doi: 10.1016/j.gene.2009.01.006

Reynoso, M. A., Blanco, F. A., Bailey-Serres, J., Crespi, M., and Zanetti, M. E. (2013). Selective recruitment of mRNAs and miRNAs to polyribosomes in response to rhizobia infection in Medicago truncatula. Plant J. 73, 289-301. doi: $10.1111 /$ tpj. 12033

Rival, A., Ilbert, P., Labeyrie, A., Torres, E., Doulbeau, S., Personne, A., et al. (2013). Variations in genomic DNA methylation during the long-term in vitro proliferation of oil palm embryogenic suspension cultures. Plant Cell Rep. 32, 359-368. doi: 10.1007/s00299-012-1369-y 
Robert-Seilaniantz, A., MacLean, D., Jikumaru, Y., Hill, L., Yamaguchi, S., Kamiya, Y., et al. (2011). The microRNA miR393 re-directs secondary metabolite biosynthesis away from camalexin and towards glucosinolates. Plant J. 67, 218-231. doi: 10.1111/j.1365-313X.2011.04591.x

Schwab, R., Palatnik, J. F., Riester, M., Schommer, C., Schmid, M., and Weigel, D. (2005). Specific effects of microRNAs on the plant transcriptome. Dev. Cell 8, 517-527. doi: 10.1016/j.devcel.2005.01.018

Smulders, M. J. M., and De Klerk, G. J. (2011). Epigenetics in plant tissue culture. Plant Growth Regul. 63, 137-146. doi: 10.1007/s10725-0109531-4

Song, G. H., Zhao, C. F., Zhang, M., Fu, C. H., Zhang, H., and Yu, L. J. (2014). Correlation analysis of the taxane core functional group modification, enzyme expression, and metabolite accumulation profiles under methyl jasmonate treatment. Biotechnol. Prog. 30, 269-280. doi: 10.1002/ btpr.1864

Sun, G. (2012). MicroRNAs and their diverse functions in plants. Plant Mol. Biol. 80, 17-36. doi: 10.1007/s11103-011-9817-6

Sunkar, R., Li, Y. F., and Jagadeeswaran, G. (2012). Functions of microRNAs in plant stress responses. Trends Plant Sci. 17, 196-203. doi: 10.1016/j.tplants.2012.01.010

Sunkar, R., and Zhu, J. K. (2004). Novel and stress-regulated microRNAs and other small RNAs from Arabidopsis. Plant Cell 16, 2001-2019. doi: $10.1105 /$ tpc. 104.022830

Vaucheret, H. (2006). Post-transcriptional small RNA pathways in plants: mechanisms and regulations. Genes Dev. 20, 759-771. doi: 10.1101/gad.1410506

Wang, Q. M., and Wang, L. (2012). An evolutionary view of plant tissue culture: somaclonal variation and selection. Plant Cell Rep. 31, 1535-1547. doi: $10.1007 / \mathrm{s} 00299-012-1281-5$
Xu, Q., Liu, Y., Zhu, A., Wu, X., Ye, J., Yu, K., et al. (2010). Discovery and comparative profiling of microRNAs in a sweet orange red-flesh mutant and its wild type. BMC Genomics 11:246. doi: 10.1186/1471-2164-11-246

Yang, K. C., Ku, Y. C., Lovett, M., and Nerbonne, J. M. (2012). Combined deep microRNA and mRNA sequencing identifies protective transcriptomal signature of enhanced PI3Kalpha signaling in cardiac hypertrophy. J. Mol. Cell. Cardiol. 53, 101-112. doi: 10.1016/j.yjmcc.2012.04.012

Zeng, H. Q., Wang, G. P., Hu, X. Y., Wang, H. Z., Du, L. Q., and Zhu, Y. Y. (2014). Role of microRNAs in plant responses to nutrient stress. Plant Soil 374, 1005-1021. doi: 10.1007/s11104-013-1907-6

Zhang, X. D., Zhang, Y. H., Ling, Y. H., Liu, Y., Cao, H. G., Yin, Z. J., et al. (2013). Characterization and differential expression of microRNAs in the ovaries of pregnant and non-pregnant goats (Capra hircus). BMC Genomics 14:157. doi: 10.1186/1471-2164-14-157

Zhao, J., and Verpoorte, R. (2007). Manipulating indole alkaloid production by Catharanthus roseus cell cultures in bioreactors: from biochemical processing to metabolic engineering. Phytochem. Rev. 6, 435-457. doi: 10.1007/s11101006-9050-0

Conflict of Interest Statement: The authors declare that the research was conducted in the absence of any commercial or financial relationships that could be construed as a potential conflict of interest.

Copyright $\odot 2015$ Zhang, Dong, Nie, Lu, Fu and Yu. This is an open-access article distributed under the terms of the Creative Commons Attribution License (CC BY). The use, distribution or reproduction in other forums is permitted, provided the original author(s) or licensor are credited and that the original publication in this journal is cited, in accordance with accepted academic practice. No use, distribution or reproduction is permitted which does not comply with these terms. 